\title{
Communications
}

\section{Examen de spécialiste \\ Examen de spécialiste en vue de l'obtention du titre de spécialiste en gastroentérologie}

Partie A: European Board of Gastroenterology and Hepatology Knowledge Examination

Date: 20 avril 2016

Lieu: à différents endroits en Suisse (Genève, Zurich, évtl. Lausanne)

Partie B: Examen pratique oral avec des discussion de cas

Date et Lieu: 2-4 semaines plus tard. Lieu et date seront communiqués en novembre 2015

Délai d'inscription: 16 décembre 2015 au 27 janvier 2016

Vous trouverez de plus amples informations sur le site web de l'ISFM www.siwf.ch $\rightarrow$ Domaines spécialisés $\rightarrow$ Titres de spécialiste et formations approfondies (formation postgraduée) $\rightarrow$ Gastroentérologie

\section{Collège de Médecine de Premier Recours CMPR}

\section{Prix CMPR de recherche en médecine de premier recours 2016*}

Spécialité: médecine de premier recours

Critères des travaux soumis: Travaux scientifiques achevés en Suisse ou par des auteurs suisses habitant à l'étranger, qui apportent une contribution importante aux soins de base offerts par le médecin de premier recours, notamment:

- à la qualité du traitement et de la prise en charge des patients de premier recours

- à la pratique médicale des médecins de premier recours (thématique valable, pertinente et réalisable en pratique)

- à la recherche sur le cabinet du médecin de premier recours: fondements, conditions de travail, gestion et préservation des ressources

\section{Prix: $30000 \mathrm{CHF}$}

Le prix sera accordé à un seul lauréat pour son travail scientifique ou alors il sera réparti sur plusieurs auteurs (sans possibilité de recours devant les tribunaux).

Participant(e)s: Auteurs en Suisse ou personnes suisses habitant à l'étranger ayant achevé un travail scientifique majeur sur la médecine de premier recours dans les trois dernières années.

\section{Délai de soumission: 1 décembre 2015}

Remise du prix: Lors des colloques de formation continue du CMPR, les 23 et 24 juin 2016 à Lucerne et le 8 septembre 2016 à Lausanne.

\footnotetext{
Sujets actuels de forum Joignez la discussion en ligne sur www.saez.ch

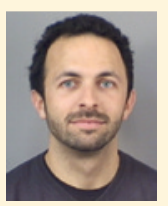

Dr med. Roman Hari,

Chef de projet formation postgraduée, SOS Ärzte, Zurich

Formation médicale postgraduée

La pénurie de médecins, une chance pour la formation postgraduée

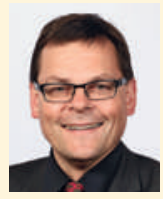

Dr med. Tarzis Jung

Président de la Société Suisse de Radiologie (SGR-SSR)

Médecin-chef de l'Institut de Radiologie et de Médecine nucléaire, Stadtspital Waid, Zurich

«Non, une IRM n'est pas toujours nécessaire.»

Réponse à la contribution sur les examens spéciaux
}

Comité: Jury indépendant, nommé par le conseil de fondation du CMPR

Information: Détail des conditions de participation: dossier à fournir sous forme électronique ou de $\mathrm{CD}$ (pas de disquettes) et sous forme papier: formulaire d'inscription (www.kollegium.ch/rd/f.html), curriculum vitae de l'auteur principal, manuscrit, brève lettre d'accompagnement «importance de ce travail pour la médecine de premier recours».

Nous ne prendrons pas en considération les travaux ayant déjà été soumis une fois et il n'y aura pas d'échange de courrier au sujet de l'attribution des prix.

Lieu de contact: Collège de médecine de premier recours CMPR, Secrétariat de la Recherche en médecine de premier recours, Rue de l'Hôpital 15, CP 1552, 1701 Fribourg, khm[at]hin.ch; www.kollegium.ch/rd/f.html

* offert par la maison Mepha Pharma SA

Société Suisse de Rhumatologie (SSR)

Prix de recherche «Jean et Linette Warnery" 2016

La Société Suisse de Rhumatologie (SSR) met au concours le prix de recherche en arthrite rhumatoïde de 60000 francs, offert par la Fondation "Jean et Linette Warnery» à Morges.

Le prix récompense un ou plusieurs travaux concernant l'arthrite rhumatoïde, effectués en Suisse ou par des Suisses travaillant à l'étranger. Il ne peut être attribué plus de deux fois au même auteur.

Peuvent être soumis des travaux publiés dès le $1^{\text {er }}$ janvier 2014 ainsi que ceux acceptés pour publication à la date de clôture des inscriptions (30 avril 2016).

Les travaux doivent être adressés jusqu'au 30 avril 2016 en deux exemplaires à:

Société Suisse de Rhumatologie, Postfach, Josefstrasse 92, 8005 Zurich

Le prix sera remis par le président de la SSR à l'occasion de l'assemblée annuelle de la SSR qui aura lieu entre le 25 et 26 août 2016 à Interlaken. Le lauréat présentera son travail dans le cadre du programme scientifique du congrès.

Renseignements: Dr O. Caviezel, président de la Fondation "Jean et Linette Warnery", 4, chemin des Emetaux, 1131 Tolochenaz. 